\title{
Quantitative evaluation of the plutonium proliferation resistance*
}

\author{
Evgeny G. Kulikov ${ }^{1}$, Gennady G. Kulikov ${ }^{1}$, Vladimir A. Apse ${ }^{1}$, Anatoly N. Shmelev ${ }^{1}$, \\ Nikolay I. Geraskin ${ }^{1}$ \\ 1 National Research Nuclear University MEPhI (Moscow Engineering Physics Institute), Kashirskoe shosse 31, Moscow, 115409, Russian \\ Federation \\ Corresponding author: Evgeny G. Kulikov (egkulikov@mephi.ru)
}

Academic editor: Georgy Tikhomirov • Received 13 August 2018 Accepted 16 November 2018 Published 7 December 2018

Citation: Kulikov EG, Kulikov GG, Apse VA, Shmelev AN, Geraskin NI (2018) Quantitative evaluation of the plutonium proliferation resistance. Nuclear Energy and Technology 4(3): 191-195. https://doi.org/10.3897/nucet.4.31859

\begin{abstract}
The mathematical model presented in (Kulikov et al. 2018) can be used for the quantitative evaluation of the plutonium proliferation resistance. This requires the warm-up process of an implosion nuclear explosive device (NED) with a different structure to be analyzed with respect to various heat removal conditions and the option to be identified in which the NED remains operational for the longest time possible. The fraction of the ${ }^{238} \mathrm{Pu}$ isotope with which, even in this case, the NED will prove to be operational only for quite a short time can be regarded as sufficient for the plutonium with such composition to be considered a proliferation resistant material.

The purpose of the paper is to evaluate in quantitative terms the content of ${ }^{238} \mathrm{Pu}$ in plutonium for ensuring its proliferation resistance and to identify the factors which influence significantly this evaluation.

The data, procedures and findings from earlier works on the topic, as well as the authors' own estimates and calculations were used for the study.

It has been shown that the important factors involved in the plutonium proliferation resistance evaluation are the NED technology level and the required NED lifetime.

Depending on the required lifetime, tougher requirements can be introduced with respect to the ${ }^{238} \mathrm{Pu}$ content both from the standpoint of low-technology and high-technology NEDs.

With a lifetime of five hours taken as the guide-mark (a NED is unlikely to be finally assembled, transported and used for such a short time), it is only plutonium containing $55 \%$ of ${ }^{238} \mathrm{Pu}$ that can be considered a proliferation resistant fissile material.
\end{abstract}

\section{Keywords}

Plutonium; plutonium-238; proliferation resistance; nuclear explosive device; explosive; cryogenic temperatures

\footnotetext{
* Russian text published: Izvestiya vuzov. Yadernaya Energetika (ISSN 0204-3327), 2018, n. 2, pp. 37-46

Copyright Kulikov EG et al. This is an open access article distributed under the terms of the Creative Commons Attribution License (CC-BY 4.0), which permits unrestricted use, distribution, and reproduction in any medium, provided the original author and source are credited.
} 


\section{Introduction}

To date, world's nuclear power industry has accumulated (and continues to accumulate) large quantities of irradiated fuel with rather a large content of plutonium (the accumulated plutonium quantity is over one thousand ton). This plutonium and its subsequent fate cause concerns for a number of reasons among which the problem of proliferation holds an important place.

Issues involved in ensuring the proliferation resistance of plutonium through the addition of the ${ }^{238} \mathrm{Pu}$ isotope are dealt with in a number of sources (Carson 1993, Massey and Schneider 1982, De Volpi 1982, Heising-Goodman 1980,Kessler 2007, Kessler and Chen 2008, Kessler 2011), the most prominent of which is a monograph by Dr. Gunter Kessler of Karlsruhe (Germany, 2011) (Kessler 2011).

At the same time, this fundamental work has a number of major drawbacks (an insufficiently well-grounded NED structure, consideration of the asymptotic temperature profile, absence of certain NED warm-up slowdown measures) with regard for the elimination of which the authors of this paper have developed a computational and theoretical model of an implosion NED.

The proliferation resistance of plutonium is quantitatively evaluated based on the developed model.

\section{Computational and theoretical model}

The geometrical spherically symmetrical model of a hypothetical implosion NED includes a central plutonium charge surrounded by layers of natural uranium (neutron reflector), aluminum, a chemical explosive (CE), and an external shell. Depending on the technology level, the NED may have different dimensions of certain layers and chemical explosives with a different heat resistance.

Assuming that the source of heat from $\alpha$-decay $\left({ }^{238} \mathrm{Pu}\right)$ in the central plutonium charge is spherically symmetrical, the temperature distribution by the NED layers can be found from a non-steady-state thermal conductivity equation

$\operatorname{div}[\lambda(r, T) \times \operatorname{grad} T(r, \tau)]+q_{V}(r)=c_{V}(r, T) \times \partial T(r, \tau) / \partial \tau$,

where $\lambda(r, T), c_{V}(r, T)$ are the thermal conductivity and the specific heat per volume of the material depending on the temperature $T(r, \tau) ; T(r, \tau)$ is the temperature at the point $r$ at the time $\tau$; and $q_{V}(r)$ is the intensity of the heat source.

The least heat-resistant component of an implosion NED is the chemical explosive. Depending on the type of the chemical explosive used in the NED, the toughest criterion that defines the serviceability of an implosion NED can be both melting and decomposition of $2 \%$ of the chemical explosive molecules. Besides, it is assumed that the melting of the fissile material (plutonium) renders the implosion NED nonoperational.

The fraction of the ${ }^{238} \mathrm{Pu}$ isotope with which the respective temperature profile becomes established, which is indicative of the implosion NED being unserviceable (plutonium melting and/or melting or decomposition of $2 \%$ of the chemical explosive molecules), can be taken as sufficient for the plutonium with such composition to be considered a proliferation resistant material.

A more detailed description of the computational and theoretical model is provided in (Kulikov et al. 2018).

To a great extent, the uncertainty of the estimates obtained using this model can be explained by the impossibility to take into account the exact structure of the implosion NED as well as by the complexity of the implosion NED final assembly and transportation time estimation.

\section{Recommendations on the proliferation resistance of plutonium: low technology level of the NED nuclear components, heat-resistant explosive}

A NED is considered in which the nuclear components are low-technology and a heat-resistant explosive (TATB (TATB - Wikipedia 2017, Mulford and Romero 1998, Arjun Singh et al. 2013, Hollowell et al. 2014)) is used in the nonnuclear components, with measures taken simultaneously to extend the NED lifetime (the time for which the device remains serviceable). This will make it possible to estimate the heat source power (and, therefore, the fraction of ${ }^{238} \mathrm{Pu}$ ) which ensures the plutonium proliferation resistance (Kulikov et al. 2010, Kulikov et al. 2009).

\section{Measures 1. Pre-cooling of the NED components}

It is evident that a pre-cooled NED will remain serviceable for a longer time (before its overheating and failure) than a NED that has not been pre-cooled if there is a fixed heat source in the fissile material.

The heat capacity of materials under cryogenic temperatures is extremely low, so there is no sense in trying to cool the NED's components to the maximum possible extent (to a cryogenic temperature) to extend the NED lifetime. It has been found that the cooling of the NED components to a temperature below that of liquid nitrogen $(77 \mathrm{~K})$ has little effect on the heat source power required for the proliferation resistance of plutonium: it grows only $3 \%$ when comparing the cases of cooling to $77 \mathrm{~K}$ and to $4 \mathrm{~K}$. Further, therefore, we shall keep in mind the case when the NED components are cooled to a temperature of not lower than $77 \mathrm{~K}$.

It should be however noted that the plutonium temperature shall not exceed $198 \mathrm{~K}$ throughout the warm-up period. The thing is that plutonium stabilized in the $\delta$-phase 
changes to the $\alpha^{\prime}$-phase at lower temperatures which is accompanied by an abrupt volume change; its change back to the $\delta$-phase is not fully reversible (Plutonium. Vol. 1. 2003, Plutonium. Vol. 2. 2003). As a result, such phase transformations can lead to the NED serviceability lost in part or in full.

We shall note that the NED is thermally isolated from the environment after the initial cooling for maintaining its cooled state to the maximum.

When considering the warm-up process of the NED materials, it is important to note two circumstances. The first one is that the NED's central part contains plutonium, uranium and aluminum in a metallic form which are characterized by rather high heat-conduction coefficients. This means that the heat released in plutonium flows rapidly into the neighboring layers, and the difference in the temperatures of these materials during warm-up turns out to be relatively small (in the limits of $100^{\circ} \mathrm{C}$ ).

The second circumstance consists in that aluminum and the chemical explosive have the highest heat storing properties among the materials forming a NED, due to which the NED lifetime extension requires primarily the cooling of exactly these materials capable to act as the accumulator of the heat released in plutonium hindering its heating in such a way.

A NED with low-technology nuclear components is more dangerous than a high-technology NED because of a thick layer of aluminum and the chemical explosive which proves these to have high heat-storing properties.

Pre-cooling of high-technology NED components may cause an approximately 50\% increase (as compared with the case of no cooling) in the heat source power required for the plutonium proliferation resistance. This, accordingly, leads to increased requirements to the content of ${ }^{238} \mathrm{Pu}$ in plutonium.

\section{Measure 2. Providing the NED with an outer heat-re- moving layer}

A nearly ideal heat removal mode (the NED outer surface temperature is always constant) can be ensured in practice by providing the NED with an outer heat-removing layer of a highly heat-conductive material or a material that experiences phase changes at the pre-cooling temperature. Actually, such a layer of a particular thickness is capable to accumulate all of the heat it receives with no temperature increase (since this heat will be spent for melting or evaporation).

We shall consider a heat-removing layer which consists of $25 \%$ of a highly heat-conducting material (aluminum) and $75 \%$ of a material that experiences phase changes at the pre-cooling temperature (liquid nitrogen).

The thickness of the heat-removing layer is selected such that it is capable to accumulate, with no temperature increase, all of the heat it receives for the preset NED warm-up period up to the time the device becomes unserviceable.
A high-technology NED with a heat-removing layer that provides for the ideal heat removal case requires an approximately $15 \%$ increase in the heat source power required for the plutonium proliferation resistance.

\section{Measure 3. Adding a heat-insulating layer to the NED structure for the purposeful temperature field redis- tribution}

It has been found by calculations that an implosion NED loses its serviceability due to excessive heating of the chemical explosive, while the internal layers (plutonium, uranium, aluminum) have temperatures which are far from being limiting for these (e.g., the melting temperature). Therefore, measures need to be taken to prevent the heat transport from the internal NED layers into the chemical explosive layer: this will extend the device's lifetime. Thus, it is possible to introduce a thin layer (e.g., $5 \mathrm{~mm}$ thick) of a material with low heat conductivity between the aluminum and chemical explosive layers.

Certain requirements exist for the heat-insulating layer material which is required to have low heat conductivity and a high working temperature. Quartz aerogel used in construction as a heat-insulating and heat-retaining material appears to be one of the most attractive materials for the heat-insulating layer (Papadopoulos 2005). Indeed, it is characterized by an extremely low heat conductivity $(0.017 \mathrm{~W} / \mathrm{m} \cdot \mathrm{K}$ in air at atmospheric pressure), while at the same time having a high working temperature (1200 ${ }^{\circ} \mathrm{C}$ ) (Aerogel - Wikipedia 2016, Gurav et al. 2010, Ayers and Hunt 2001, Parvathy et al. 2008).

Adding a heat-insulating layer to the structure of a high-technology NED for the purposeful temperature field redistribution leads to a more than $50 \%$ increase in the heat source power required for the plutonium proliferation resistance.

Thus, it can be expected that all of the above measures contributing to the NED lifetime extension will more than double, if taken, the required heat source power.

Monograph (Kessler 2011) considers a case when plutonium is initially cooled to $73 \mathrm{~K}$, and the rest of the materials composing the device have an indoor temperature. A conclusion is made that, where plutonium is the heat source with a power of $0.48 \mathrm{~kW}$, the plutonium melting is observed as soon as after seven hours.

However, (Kessler 2011) does not consider the cooling of the other NED materials (uranium, aluminum, chemical explosive). Among the NED materials, the highest heat-storing properties are possessed by aluminum and the chemical explosive due to which a case should be considered where these materials are pre-cooled.

Also, such pre-cooling of the NED components shall be selected that the plutonium temperature is over $198 \mathrm{~K}$ throughout the warm-up process. It has been found by calculations that a NED with low-technology nuclear components and nonnuclear components containing a heat-resistant explosive (TATB) is capable to remain serviceable for the longest time possible (with the plutonium tem- 
perature being always above $198 \mathrm{~K}$ ) with the following initial temperature field: plutonium and uranium have a temperature of $300 \mathrm{~K}$, aluminum has a temperature of 150 $\mathrm{K}$, and the chemical explosive (TATB) has a temperature of $77 \mathrm{~K}$. In this case, the melting of plutonium containing the $\alpha$-decay heat source of $0.48 \mathrm{~kW}$ is observed as soon as after seven days (!).

It has been therefore shown that the aluminum and chemical explosive cooling contributes much more to the NED lifetime extension than the plutonium cooling.

A time span of about seven days may turn out to be sufficient for the final NED assembly stage and for its further transportation and use. With a lifetime of five hours used as the guide-mark (a NED is unlikely to be finally assembled, transported and used for such a short time), a heat source of about $4 \mathrm{~kW}$ is required. This heat source can be created by adding $55 \%$ of ${ }^{238} \mathrm{Pu}$ to plutonium.

This estimate differs greatly from what is stated in (Kessler 2011). The author suggests that the threshold states are capable to use technologies of low to medium levels rather than a high NED technology. Estimates for the low-tech NED options show that the explosive starts to melt with the ${ }^{238} \mathrm{Pu}$ content of only $1.8 \%$, that is, in other words, the explosive layer's heterogeneous structure in the NED breaks down and the device is rendered unserviceable. Such content of ${ }^{238} \mathrm{Pu}$ in reactor-grade plutonium has already been achieved and has been exceeded by now in the nuclear power fuel cycle with light-water reactors using $\mathrm{UO}_{2}$ fuel with a burn-up of 35 $\mathrm{GW} \cdot$ day/t.

This was the basis for Dr. G. Kessler's conclusion that the existing (U-Pu) cycle can be considered proliferation resistant for the threshold states he believes capable of building a NED in a range of low to medium technology levels (Kessler 2011).

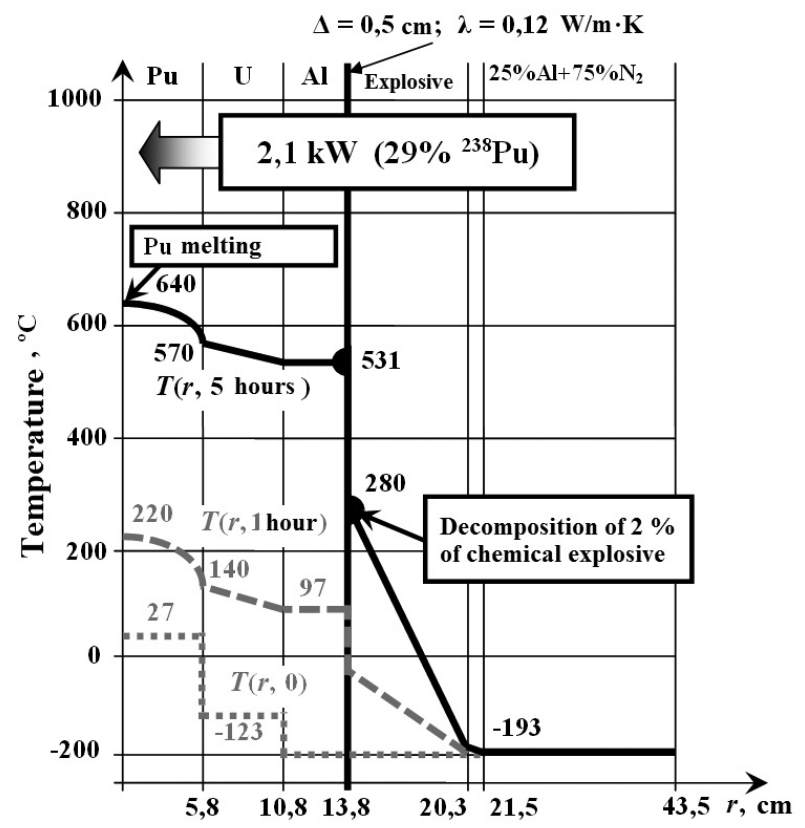

Figure 1.Temperature profiles in a high-technology implosion NED with all NED lifetime extension measures used.

\section{Recommendations on the proliferation resistance of plutonium: high technology level of the NED nuclear components, heat-resistant explosive}

A NED is considered in which the nuclear components are high-technology and a heat-resistant explosive (TATB) is used in the nonnuclear components, with, as earlier, all of the above measures taken simultaneously to extend the device's lifetime. This will make it possible to estimate the heat source power (and, therefore, the ${ }^{238} \mathrm{Pu}$ fraction) that ensures the plutonium proliferation resistance.

Such pre-cooling of the NED components shall be selected that the plutonium temperature is over $198 \mathrm{~K}$ throughout the warm-up process. It has been found by calculations that a NED with high-tech nuclear components and nonnuclear components containing a heat-resistant explosive (TATB) is capable to remain serviceable for the longest time possible (with the plutonium temperature being always above $198 \mathrm{~K}$ ) with the following initial temperature field: plutonium has a temperature of 300 $\mathrm{K}$, uranium has a temperature of $150 \mathrm{~K}$, and aluminum and the chemical explosive have a temperature of $77 \mathrm{~K}$.

To estimate the heat source power (the ${ }^{238} \mathrm{Pu}$ fraction) that ensures the plutonium proliferation resistance, it is required to fix the NED lifetime. A lifetime of five hours will be taken as an example: a NED is unlikely to be finally assembled, transported and used for such a short time. Fig. 1 presents temperature profiles in a high-technology implosion NED at the times 0,1 and $5 \mathrm{~h}$.

Based on the selected criterion for the proliferation resistance of fissile materials (the serviceability of a NED based on these shall be not less than five hours), we conclude that the required heat source power is $2.1 \mathrm{~kW}$ (Fig. $1)$. In this case, it is only plutonium containing at least $29 \%$ of ${ }^{238} \mathrm{Pu}$ that may be considered a proliferation resistant material.

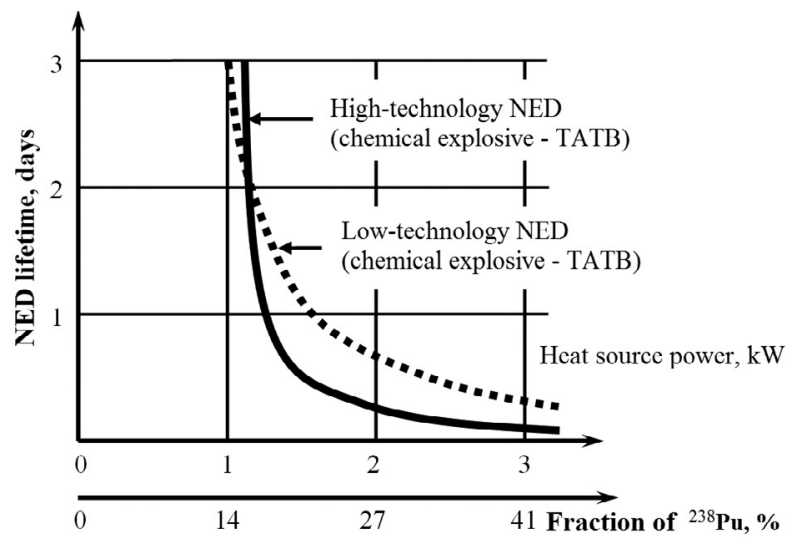

Figure 2. NED lifetime as a function of $\alpha$-decay heat source power $\left({ }^{238} \mathrm{Pu}\right.$ fraction in plutonium). 
Since the time for the implosion NED final assembly and transportation is difficult to estimate, it is required to consider a broad range of values for the lifetime which is the key factor for evaluating the proliferation resistance of fissile materials (Fig. 2).

In conditions of no accurate data available on the time required for the NED final assembly and transportation, no firm conclusion can be made on the content of ${ }^{238} \mathrm{Pu}$ required for the proliferation resistance of plutonium. It follows from Fig. 2 that the required ${ }^{238} \mathrm{Pu}$ content shall not exceed at least $14 \%$. According to the IAEA recommendations, plutonium containing over $80 \%$ of ${ }^{238} \mathrm{Pu}$ can be considered proliferation resistant. The investigated mathematical model provides for approximately the same result with a lifetime of about two hours.

The uncertainty of the estimate for the required content of ${ }^{238} \mathrm{Pu}$ which ensures the plutonium proliferation resistance is explained by the uncertainty of the time needed for the NED final assembly and transportation. Depending on this, the required ${ }^{238} \mathrm{Pu}$ content is from $14 \%$ (the
NED assembly and transportation time is several days to several weeks) to $80 \%$ (the NED assembly and transportation time is two hours).

\section{Conclusion}

The proliferation resistance of plutonium has been quantitatively evaluated based on a developed computational and theoretical model. It has been shown that important factors are the NED technology level and the required lifetime of the device.

It must be stressed that one cannot make a conclusion as to the proliferation resistance of plutonium based on considering only a low-technology NED or only a high-technology NED. Depending on the required lifetime, there may be tougher requirements to the content of ${ }^{238} \mathrm{Pu}$ introduced with respect to high-technology NEDs (a lifetime of over two days) and with respect to low-technology NEDs (a lifetime of less than two days) (see Fig. 2).

\section{References}

- Aerogel - Wikipedia, the free encyclopedia (2016) Available at: https://en.wikipedia.org/wiki/Aerogel [accessed 22 Oct. 2016].

- Singh A, Kumar M, Soni P, Singh M, Srivastava A (2013) Mechanical and Explosive Properties of Plastic Bonded Explosives Based on Mixture of HMX and TATB. Defence Science Journal, 63(6): 622629. https://doi.org/10.14429/dsj.63.5764

- Ayers MR, Hunt AJ (2001) Synthesis and properties of chitosan-silica hybrid aerogels. Journal of Non-Crystalline Solids, 1-3: 123-127.

- Carson MJ (1993) Explosive Properties of Reactor-Grade Plutonium. Science \& Global Security, 4: 111-128.

- De Volpi A (1982) Denaturing Fissile Materials. Progress in Nuclear Energy, 10 (2): 161-220. https://doi.org/10.1016/01491970(82)90022-1

- Heising-Goodman CD (1980) An Evaluation of the Plutonium Denaturing Concept as an Effective Safeguards Method. Nuclear Technology, 50: 242-251. https://doi.org/10.13182/NT80-A32527

- Hollowell BC, Gustavsen RL, Dattelbaum DM, Bartram BD (2014) Shock initiation of the TATB-based explosive PBX 9502 cooled to 77 Kelvin. Journal of Physics Conference Series, 500 (18).

- IAEA Safeguards Glossary (2002) 2001 Edition. International Nuclear Verification Series No. 3. Vienna. International Atomic Energy Agency.

- Gurav JL, Jung I-K, Park H-H, Kang ES, Nadargi DY (2010) Silica Aerogel: Synthesis and Applications. Hindawi Publishing Corporation, Journal of Nanomaterials, 2010, 11 pp. Article ID 409310 https://doi.org/10.1155/2010/409310

- Kessler G (2007) Plutonium Denaturing by ${ }^{238} \mathrm{Pu}$. Nuclear Science and Engineering, 155: 53-73. https://doi.org/10.13182/NSE07-A2644

- Kessler G (2011) Proliferation-Proof Uranium/Plutonium Fuel Cycles. Safeguards and Non-Proliferation. Germany. KIT Scientific Publishing.

- Kessler G, Chen X-N (2008) Thermal Analysis of Hypothetical Nuclear Explosive Devices Containing Reactor-grade Plutonium with
Higher Content of Pu-238. Obninsk Workshop, 29 September - 3 October 2008.

- Kulikov EG, Kulikov GG, Apse VA, Shmelev AN, Geraskin NI (2018) A computational model and physical and technical factors that define the proliferation resistance of plutonium. Izvestiya vuzov. Yadernaya energetika, 1: 23-32.

- Kulikov Ye, Shmelev A, Apse V, Kulikov G (2009) Mathematical models for quantitative evaluation of fissionable materials proliferation protection. American Nuclear Society - the $4^{\text {th }}$ Topical Meeting on Advances in Nuclear Fuel Management 2009 (ANFM IV). Hilton Head Island, South Carolina, USA, April 12-15.

- Kulikov Ye, Shmelev A, Apse V, Kulikov G (2010) Computational models for the quantitative evaluation of proliferation resistance for fissionable materials. Izvestiya vysshykh uchebnykh zavedeny. Yadernaya energetika, 2: 184-195.

- Massey JV, Schneider A (1982) The Role of Plutonium-238 in Nuclear Fuel Cycles. Nuclear Technology, 56.

- Mulford RN, Romero JA (1998) Sensitivity of the TATB-based explosive PBX-9502 after thermal expansion. AIP Conf. Proc. 429.

- Papadopoulos AM (2005) State of the art in thermal insulation materials and aims for future developments. Energy and Buildings, 37: 77-86. https://doi.org/10.1016/j.enbuild.2004.05.006

- Parvathy Rao A., Venkateswara Rao A, Bangi U(2008) Low thermal-conductive, transparent and hydrophobic ambient pressure dried silica aerogels with various preparation conditions using sodium silicate solutions. Journal of Sol-Gel Science and Technology, 1: 85-94.

- Plutonium. Fundamental problems. Vol. 1. (2003) Sarov. RFNC-VNIIEF Publ.

- Plutonium. Fundamental problems. Vol. 2. (2003) Sarov. RFNC-VNIIEF Publ.

- TATB - Wikipedia, the free encyclopedia (2017) Available at: https://en.wikipedia.org/wiki/TATB [accessed 27 Oct. 2017]. 